\title{
Editorial
}

\section{Joachim Bill}

Institute for Materials Science, University of Stuttgart, Stuttgart, Germany

\section{Giulia Santomauro}

Institute for Materials Science, University of Stuttgart, Stuttgart, Germany
Inorganic functional materials have tremendous impact on key technologies relevant for the further development of future fields like information technology or energy generation and storage. In this connection, complex-structured multifunctional inorganic materials as well as their hybrids with organic components play a main role. The generation of such materials with defined structure and stoichiometry via conventional processing is limited, since such processes require increased temperatures and/or pressures as well as large technological efforts. Accordingly, there are world-wide research activities trying to overcome these limitations and searching for new procedures, which allow the manufacturing of new materials at ambient conditions with reduced processing efforts.

Living nature provides impressive evolution-optimized processes, which lead to complex-structured multifunctional inorganic solids. The formation of these inorganic materials occurs via biomineralization in aqueous environments at ambient conditions and is genetically determined. During these processes biopolymeric templates that control the mineralization and the structure formation of the inorganic components play a crucial role. The biomineralization processes also involve molecular selfassembly and finally yield composites made of non-metallic inorganic solids like calcium phosphate or calcium carbonate and bioorganic components. Such inorganic/bioorganic hybrids exhibit unique multifunctional features. Their performance and property spectrum is tuned and expanded by the incorporation of the bioorganic fraction. Even though many of the technically relevant materials cannot be generated by the processes developed through biological evolution, the research on biomineralization principles provides promising perspectives for the generation of inorganic functional materials via the interaction between bioorganic and inorganic components. Knowing the principles of in vivo biomineralization of living organisms also offers the possibility of their transfer to in vitro procedures with non-biogenic elements, leading to technically applicable materials.

This themed issue, entitled "DFG Priority Program 1569 Generation of Multifunctional Inorganic Materials by Molecular Bionics" is dedicated to the transfer of the principles of biomineralization to the generation of complex-structured multifunctional inorganic materials as well as of their hybrids with bioorganic components. It comprises work carried out within the scope of the correspondent Priority Program (Schwerpunktprogramm, SPP) 1569 of the Deutsche Forschungsgemeinschaft (DFG), where the following research items were addressed.

- The in vitro and in vivo generation of such materials directed by biomolecule-based templates with a main focus on $2 \mathrm{D}$ and 3D structures.

- The characterization of the formation mechanisms as well as of the structure of the materials.

- The investigation and design of the physical and chemical properties of the materials.

- The computational modeling of the formation, structure and properties of the materials.

The issue provides an overview about the topics of the involved research projects. It also contains further reading and literature on the results obtained within the program.

For the comprehensive treatment of the aforementioned topics a strongly interdisciplinary base is indispensable. This base and frame was provided by the DFG through the establishment of the SPP 1569 , which is highly acknowledged by all participants of this program. Special thanks also to the members of the review panel and to Dr. Burkhard Jahnen (DFG) for their excellent support during the whole funding period of the program for six years. 